\title{
Theoretical Study of Hydrogen Adsorption on Ruthenium Clusters
}

\author{
Gui-Xian Ge · Hong-Xia Yan • Qun Jing • \\ You-Hua Luo
}

Received: 4 May 2011/Published online: 22 June 2011

(C) The Author(s) 2011. This article is published with open access at Springerlink.com

\begin{abstract}
The geometries, stabilities, electronic, and magnetic properties of hydrogen adsorption on $\mathrm{Ru}_{n}$ clusters have been systematically investigated by using density functional theory with generalized gradient approximation. The result indicates the absorbed species does not lead to a rearrangement of the basic cluster. For $n>2$, three different adsorption patterns are found for the $\mathrm{Ru}_{n} \mathrm{H}_{2}$ complexes: One $\mathrm{H}$ atom binds to the $\mathrm{Ru}$ top site, and another $\mathrm{H}$ binds to the bridge site for $n=3,5,6,8$; bridge site adsorption for $n=4$; hollow site and top site adsorption for $n=7$. The adsorption energies display oscillation and reach the peak at $n=2$, 4,7 , implying their high chemical reactivity. The small electron transferred number between $\mathrm{H}$ atoms and $\mathrm{Ru}_{n}$ clusters indicates that the interaction between $\mathrm{H}$ atoms and $\mathrm{Ru}_{n}$ clusters is small. When $\mathrm{H}_{2}$ is absorbed on the $\mathrm{Ru}_{n}$ clusters, the chemical activity of corresponding clusters is dramatically increased. The absorbed $\mathrm{H}_{2}$ can lead to an oscillatory behavior of the magnetic moments, and this behavior is rooted in the electronic structure of the preceding cluster and the changes in the magnetic moment are indicative of the relative ordering of the majority and minority LUMO's. The second order difference indicates 5 is magic number in $\mathrm{Ru}_{n} \mathrm{H}_{2}$ and $\mathrm{Ru}_{n}$ clusters.
\end{abstract}

Keywords $\mathrm{Ru}_{n} \mathrm{H}_{2}$ cluster - Electronic properties · Equilibrium geometries

G.-X. Ge $(\bowtie) \cdot$ H.-X. Yan · Q. Jing

Key Laboratory of Ecophysics and Department of Physics, School of Sciences, Shihezi University, Xinjiang 832003, China

e-mail: geguixian@126.com

Y.-H. Luo

Department of Physics, East China University of Science and Technology, Shanghai 200237, China

Y.-H. Luo

Institute for Theoretical Physics, Henan University, Kaifeng 475001, China 


\section{Introduction}

In the past 20 years, small metal clusters have been used to model the adsorption of different molecules with the purpose to obtain a good description of the activity of many metal catalysts. Due to the small particles size of a well dispersed catalysts, most or almost all of its atom are surface atoms, so they exhibit structural and reaction properties different from those of the bulk, they are appropriate to surface reactions as the most process in catalysis are. Many previous researches showed that the catalytic properties of clusters are structure dependent, leading to different adsorption energies and sites for different structures of clusters [1-6]. Hydrogen is employed in many modern technologies [7-21], so surface reactions of hydrogen molecules in transition metals have been carried out over the adsorption and desorption of hydrogen and deuterium molecules on well-defined single crystal metals. Axel Pramann et al. [22] studied hydrogen chemisorption rates and electronic structures of small $\mathrm{Nb}_{n} \mathrm{Al}^{-}$clusters by photoelectron spectroscopic method. Poulain et al. [23] studied the $\mathrm{C}_{2 v}$ and $\mathrm{C}_{3 v}$ reaction of $\mathrm{H}_{2}$ with a $\mathrm{Pt}_{4}$ cluster. Hege Strmsnes et al. [24] studied the chemisorption of molecular hydrogen on a seven atom gold cluster using explicitly correlated wave functions. On the other hand, an area that has attracted recent attention is the effect of chemisorption on the magnetic properties of clusters. For example, Whetten et al. [25] studied the reactivity of $\mathrm{Fe}_{n}$ clusters toward $\mathrm{H}_{2}$ and $\mathrm{D}_{2}$, and found the Fermi energy and the band filling could be modulated by adding hydrogen suggests that one could expect interesting effects on magnetic properties. Indeed, Knickelbein et al. [26] recently investigated the effect of hydrogen on the magnetic moment of $\mathrm{Fe}_{n}$ clusters in molecular beam experiments and found intriguing results. Knickelbein et al. generated $\mathrm{Fe}_{n}$ clusters containing 10-25 atoms in molecular beams and the clusters were saturated with hydrogen. The hydrogenated clusters were subsequently passed through the Stern Gerlach gradient fields. They found that unlike the case of larger nanoparticles and thin films where the hydrogen adsorption quenches the magnetic moment [27], the magnetic moments of the saturated hydrogenated clusters containing 12-25 atoms were higher than those of the free clusters.

Ruthenium is the main element used for hydrogenation and dehydrogenation catalytic reactions. Liu et al. [28] studied adsorption characteristics of atomic nitrogen on ruthenium surfaces, they found that atomic nitrogen always preferably occupies the high coordination sites on Ru surfaces. Carmelo Crisafulli et al. [29] have studied Ni-Ru bimetallic catalysts for the $\mathrm{CO}_{2}$ reforming of methane. Romain Berthoud et al. [30] have studied hydrogen and oxygen adsorption stoichiometries on silica supported ruthenium nanoparticles. Zheng et al. [31] have investigated $\mathrm{NH}_{3}$ decomposition kinetics on supported $\mathrm{Ru}$ clusters. Tu et al. [32] have studied the interaction of oxygen with ruthenium clusters by density functional study. SussFink et al. [33] investigated the cluster dication $\left[\mathrm{H}_{6} \mathrm{Ru}_{4}\left(\mathrm{C}_{6} \mathrm{H}_{6}\right)_{4}\right]^{2+}$ using the a lowtemperature ${ }^{1} \mathrm{H}-\mathrm{NMR}$ and density functional theory (DFT) calculations, and found the cluster dication $\left[\mathrm{H}_{6} \mathrm{Ru}_{4}\left(\mathrm{C}_{6} \mathrm{H}_{6}\right)_{4}\right]^{2+}(1)$ tends to loose molecular hydrogen to form the cluster dication $\left[\mathrm{H}_{4} \mathrm{Ru}_{4}\left(\mathrm{C}_{6} \mathrm{H}_{6}\right)_{4}\right]^{2+}(2)$. The equilibrium between $\mathbf{1}$ and 2 can be used for catalytic hydrogenation reactions. Adams et al. investigated the Activation of hydrogen by mixed transitional metal cluster complex [34-37]. Poteau et al. [38] 
studied spectroscopic and thermodynamic properties of surfacic hydrides on $\mathrm{Ru}$ (0001) model surface: the influence of the coordination modes and the coverage by density functional theory and this study partially opens the route to DFT studies of multistep hydrogenation reactions at the surface of ruthenium nanoparticles monitored by spectroscopic techniques. Torsten Gutmann et al. [39] studied hydrido-ruthenium cluster complexes as models for reactive surface hydrogen species of ruthenium nanoparticles by solid-state ${ }^{2} \mathrm{H}$ NMR and quantum chemical calculations and found that the ${ }^{2} \mathrm{H}$ nuclear quadrupolar interaction is a sensitive tool for distinguishing the binding state of the deuterons to the transition metal.

To our knowledge, the study on the adsorption properties of small molecule on ruthenium clusters is still lacking either experimentally or theoretically so far. It would be interesting to know the adsorption ability of ruthenium clusters to small molecules such as the $\mathrm{H}_{2}$ molecule. More precisely, where are the optimal adsorption sites of ruthenium clusters to $\mathrm{H}_{2}$ molecule? Moreover, how does the adsorption of $\mathrm{H}_{2}$ molecule affect the magnetism of the clusters? To answer these questions, we have performed density functional theory (DFT) computations to explore the adsorption behavior of $\mathrm{H}_{2}$ molecule on $\mathrm{Ru}_{n}$ clusters. The purposes of this paper include the following: (a) Locate the optimal adsorption sites and (b) analyze the size-dependent structural, electronic, and magnetic properties of the $\mathrm{Ru}_{n} \mathrm{H}_{2}$ complexes, and compare with the bare ruthenium clusters.

\section{Computational Methods}

Full geometry optimizations were performed using the spin-polarized density functional theory (DFT) implemented in a DMOL package [40]. In the electronic structure calculations, all electron treatment and double numerical basis including $d$-polarization function (DNP) [40] were chosen. The exchange-correlation interaction was treated within the generalized gradient approximation (GGA) using PBE functional. Self-consistent field calculations were done with a convergence criterion of $10^{-5}$ Hartree on the total energy. The density mixing criterion for charge and spin were 0.2 and 0.5 , respectively. The Direct Inversion in an Iterative Subspace (DIIS) approach was used to speed up SCF convergence. A 0.001 Hartree of smearing was applied to the orbital occupation. In the geometry optimization, the converged thresholds were set to 0.004 Hartree/ $\AA$ for the forces, $0.005 \AA$ for the displacement and $10^{-5}$ Hartree for the energy change. Harmonic vibrational frequencies were calculated for the promising stationary points from a direct structural optimization; if an imaginary vibrational mode was found, a relaxation along coordinates of imaginary vibrational mode was carried out until the true local minimum was actually obtained. Therefore, all isomers for each cluster are guaranteed as the local minima. The on-site charges and magnetic moment were evaluated via Mulliken population analysis [41].

To test the accuracy of the theoretical method, we have calculated the dimers for $\mathrm{Ru}_{2}$ and $\mathrm{H}_{2}$ by using different functionals. For $\mathrm{Ru}_{2}$, the bond length $r=2.26 \AA$, $\omega=364.46 \mathrm{~cm}^{-1}$; the calculated results using PBE functional are in agreement with the previous theory values obtained by all-electron calculation $(r=2.41 \AA$, 
$\omega=380 \mathrm{~cm}^{-1}$ ) [42] and experimental value by Resonance Raman matrix isolation studies of mass-selected (347.1(9) $\mathrm{cm}^{-1}$ ) [43]. For $\mathrm{H}_{2}$, the bond length $r=0.748 \AA$, binding energy $E_{b}=4.665 \mathrm{eV}, \omega=4410.896 \mathrm{~cm}^{-1}$. The calculated results using PBE functional are in good agreement with experiment values $(r=0.741 \AA$, $E_{b}=4.519 \mathrm{eV} \omega=4401.21 \mathrm{~cm}^{-1}$ ) [44]. It indicates that the employed PBE scheme is reliable for the dimer $\mathrm{Ru}_{2}$ and $\mathrm{H}_{2}$. Consequently, the PBE functional are reliable and accurate enough to be applied to describe the properties of the $\mathrm{Ru}_{n} \mathrm{H}_{2}$ in this paper.

\section{Results and Discussions}

\section{Equilibrium Structures}

The low-lying isomers of $\mathrm{Ru}_{n} \mathrm{H}_{2}$ complexes and their naked counterparts $\mathrm{Ru}_{n}$ are displayed in Figs. 1 and 2. The structure and the bond length of the lowest energy structures for $\mathrm{Ru}_{n}$ and $\mathrm{Ru}_{n} \mathrm{H}_{2}$ are given in Table 1 . Here we only present the longest and the shortest interatomic distances between two Ru atoms, two $\mathrm{H}$ atoms and $\mathrm{Ru}-\mathrm{H}$ in $\mathrm{Ru}_{n} \mathrm{H}_{2}$ and in the corresponding bare $\mathrm{Ru}_{n}$. The geometries of $\mathrm{Ru}_{n} \mathrm{H}_{2}$ and $\mathrm{Ru}_{n}$ clusters with considered spin configuration are performed by DFT. The lowest energy geometry of $\mathrm{Ru}_{2}$ is a linear structure. For $\mathrm{Ru}_{3}$, the result is different from those of empirical methods by $\mathrm{Tu}$ et al. [45] who have all concluded that the structure of $\mathrm{Ru}_{3}$ is an equilateral triangle. The lowest energy structure we obtained is a triangle $\left(\mathrm{C}_{s}\right)$ whose binding energy is $0.819 \mathrm{eV}$ lower than that of equilateral structure. The lowest energy geometry of $\mathrm{Ru}_{4}$ is a quadrangle structure with $\mathrm{D}_{4 h}$ symmetry. The distorted tetrahedron is $0.348 \mathrm{eV}$ higher than that of the lowest energy geometry. For $\mathrm{Ru}_{5}$, the lowest energy structure is a square pyramid. The trigonal bi-pyramid is an isomer with $0.853 \mathrm{eV}$ higher than the square pyramid. A trigonal prism is the lowest energy structure of $\mathrm{Ru}_{6}$ cluster. The square bipyramid is $0.358 \mathrm{eV}$ higher than that of trigonal prism. The lowest energy structure of $\mathrm{Ru}_{7}$ is a twin square pyramid. The trigonal prism structure face capped a $\mathrm{Ru}$ atom is a matastable isomer, it's energy is $0.0269 \mathrm{eV}$ higher than that of the twin square pyramid. A square prism is the lowest energy structure of $\mathrm{Ru}_{8}$ cluster. Most of lowest energy structures we obtained are similar to the result obtained by Tu et al. [45].

For $\mathrm{RuH}_{2}$, the ground state corresponds to a $\mathrm{H}-\mathrm{H}$ distance of $2.428 \AA$. The $\mathrm{H}$ molecular is therefore activated. For $\mathrm{Ru}_{2}$, the additional hydrogen molecular occupies the on-top site. The $\mathrm{Ru}-\mathrm{Ru}$ bond length $\left(\mathrm{R}_{\mathrm{Ru}-\mathrm{Ru}}\right)$ is $2.322 \mathrm{~A}, 4.31 \%$ greater than that in the bare $\mathrm{Ru}_{2}$ dimer. The two $\mathrm{Ru}-\mathrm{H}$ bond lengths are 1.573 and $1.574 \AA$, respectively. Two $\mathrm{Ru}-\mathrm{Ru}-\mathrm{H}$ angles are both around 90 degree. The $\mathrm{H}-\mathrm{H}$ bond length $\left(\mathrm{R}_{\mathrm{H}-\mathrm{H}}\right)$ is $2.173 \AA$, again indicates that the $\mathrm{H}_{2}$ is activated compared with free $\mathrm{H}_{2}$ molecular.

For $n>2$, three different adsorption patterns are found for the $\mathrm{Ru}_{n} \mathrm{H}_{2}$ complexes: one $\mathrm{H}$ is absorbed on the $\mathrm{Ru}$ top site, and another $\mathrm{H}$ is absorbed on the bridge site which $\mathrm{H}$ atom sits over the $\mathrm{Ru}-\mathrm{Ru}$ bond for $n=3,5,6,8$; bridge site adsorption for $n=4$; hollow site and top site adsorption for $n=7$. The longest $\mathrm{R}_{\mathrm{Ru}-\mathrm{Ru}}$ bond 


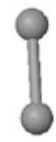

$\mathrm{Ru}_{2} \mathrm{D}_{\mathrm{h}}$ $\Delta E=0(1)$

$$
\mathrm{Ru}_{3} \mathrm{C}_{\mathrm{s}}
$$$$
\Delta E=0(7)
$$

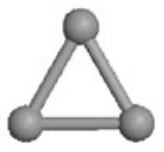

$\mathrm{Ru}_{3} \mathrm{D}_{3 \mathrm{~h}}$ $\Delta E=0.819(3)$

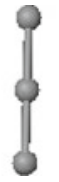

$\mathrm{Ru}_{3} \mathrm{C}_{\mathrm{v}}$ $\Delta E=0.954(3)$

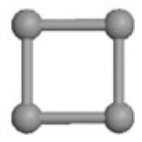

$\mathrm{Ru}_{4} \mathrm{D}_{4 \mathrm{~h}}$ $\Delta E=0(1)$

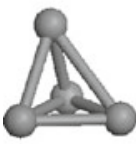

$\mathrm{Ru}_{4} \mathrm{C}_{1}$

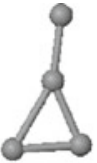

$\mathrm{Ru}_{4} \mathrm{C}_{\mathrm{s}}$

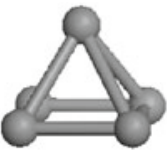

$\mathrm{Ru}_{5} \mathrm{C}_{2 \mathrm{v}}$

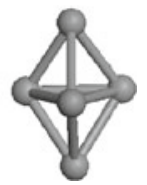

$\mathrm{Ru}_{5} \mathrm{D}_{3 \mathrm{~h}}$

$\Delta E=0.348(1)$

$\Delta E=1.682(5)$

$\Delta E=0(1)$

$\Delta E=0.853(3)$

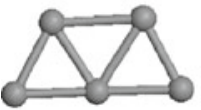

$\mathrm{Ru}_{5} \mathrm{C}_{1}$ $\Delta E=2.178(1)$

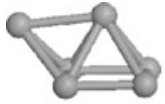

$\mathrm{Ru}_{6} \mathrm{C}_{1}$ $\Delta E=0.682(3)$

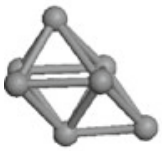

$\mathrm{Ru}_{7} \mathrm{C}_{1}$ $\Delta E=0.221(3)$

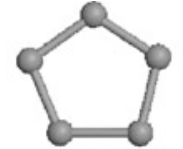

$\mathrm{Ru}_{5} \mathrm{C}_{\mathrm{s}}$ $\Delta E=2.612(1)$

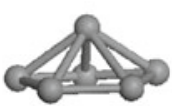

$\mathrm{Ru}_{6} \mathrm{C}_{1}$ $\Delta E=1.357$ (3)

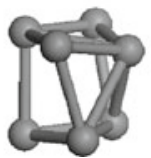

$\mathrm{Ru}_{7} \mathrm{C}_{\mathrm{s}}$ $\Delta E=0.261(3)$

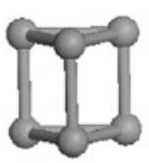

$\mathrm{Ru}_{6} \mathrm{C}_{1}$ $\Delta E=0(3)$

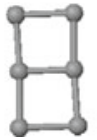

$\mathrm{Ru}_{6} \mathrm{D}_{2 \mathrm{~h}}$ $\Delta E=2.190$ (1)

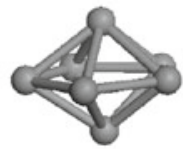

$\mathrm{Ru}_{7} \mathrm{C}_{1}$ $\Delta E=0.623$ (3)

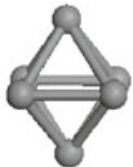

$\mathrm{Ru}_{6} \mathrm{C}_{1}$ $\Delta E=0.358(9)$

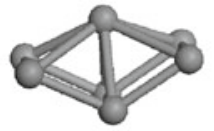

$\mathrm{Ru}_{7} \mathrm{C}_{\mathrm{s}}$ $\Delta E=0(1)$

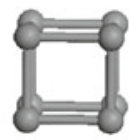

$\mathrm{Ru}_{8} \mathrm{D}_{3 \mathrm{~d}}$ $\Delta E=0(5)$

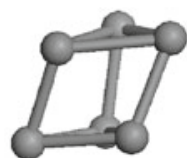

$\mathrm{Ru}_{6} \mathrm{C}_{1}$ $\Delta E=0.597(3)$

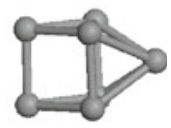

$\mathrm{Ru}_{7} \mathrm{C}_{1}$ $\Delta E=0.0269(5)$

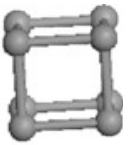

$\mathrm{Ru}_{8} \mathrm{D}_{2 \mathrm{~h}}$ $\Delta E=0.154$ (3)

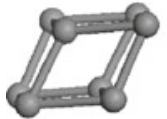

$\mathrm{Ru}_{8} \mathrm{C}_{2 \mathrm{~h}}$ $\Delta E=2.625(5)$

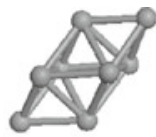

$\mathrm{Ru}_{8} \mathrm{C}_{1}$ $\Delta E=2.602$ (1)

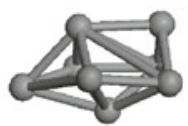

$\mathrm{Ru}_{8} \mathrm{C}_{1}$ $\Delta E=2.689$ (1)

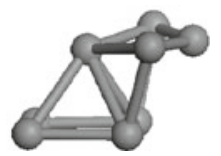

$\mathrm{Ru}_{8} \mathrm{C}_{1}$ $\Delta E=2.833$ (5)

Fig. 1 Ground state and some matastable geometries of $\mathrm{Ru}_{n}$ clusters. $\Delta E(\mathrm{eV})$ is the excess energy of an isomer as compared to the energy of the most stable one. The multiplicity is given in parentheses 


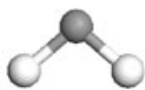

$\mathrm{RuH}_{2}$ (a)

$\Delta E=0(3)$

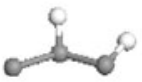

$\mathrm{Ru}_{3} \mathrm{H}_{2}$ (c)

$\Delta E=0.506(1)$

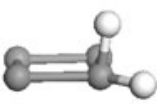

$\mathrm{Ru}_{4} \mathrm{H}_{2}$ (b)

$\Delta E=0.126(1)$

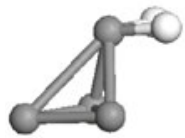

$\mathrm{Ru}_{4} \mathrm{H}_{2}(\mathrm{~g})$

$\Delta E=0.280(5)$

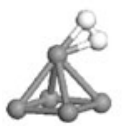

$\mathrm{Ru}_{5} \mathrm{H}_{2}(\mathrm{~b})$

$\Delta E=0.293$ (1)

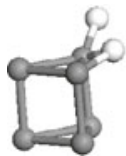

$\mathrm{Ru}_{6} \mathrm{H}_{2}$ (a)

$\Delta E=0(3)$

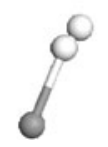

$\mathrm{RuH}_{2}(\mathrm{~b})$

$\Delta E=1.268$ (5)

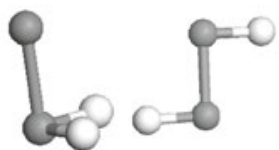

$\mathrm{Ru}_{2} \mathrm{H}_{2}$ (a)

$\Delta E=0(5)$
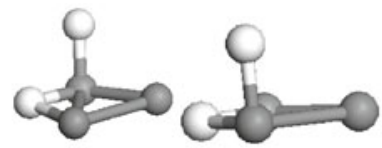

$\mathrm{Ru}_{3} \mathrm{H}_{2}$ (a) $\quad \mathrm{Ru}_{3} \mathrm{H}_{2}$ (b)

$\Delta E=0(3)$

$\Delta E=0.0862(3)$

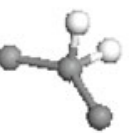

$$
\mathrm{Ru}_{3} \mathrm{H}_{2}(\mathrm{f})
$$

$\mathrm{Ru}_{4} \mathrm{H}_{2}$ (a)

$\Delta E=0.810(5)$

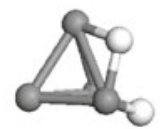

$\Delta E=0(3)$

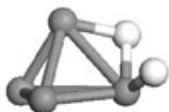

$\mathrm{Ru}_{4} \mathrm{H}_{2}(\mathrm{e})$

$\Delta E=0.172(3)$

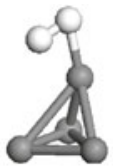

$$
\mathrm{Ru}_{4} \mathrm{H}_{2}(\mathrm{j})
$$

$\Delta E=0.925$ (3)

$\Delta E=0.511(3)$

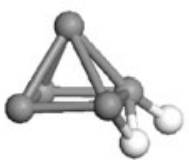

$\mathrm{Ru}_{5} \mathrm{H}_{2}(\mathrm{~d})$

$\Delta E=0.457$ (3)

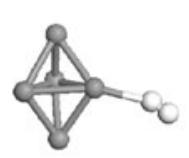

$\mathrm{Ru}_{5} \mathrm{H}_{2}(\mathrm{e})$

$\Delta E=1.569$ (3)

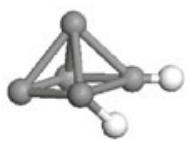

$\mathrm{Ru}_{5} \mathrm{H}_{2}$ (a)

$\Delta E=0(1)$

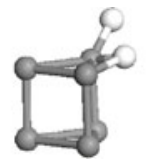

$\mathrm{Ru}_{6} \mathrm{H}_{2}$ (b)

$\Delta E=0.0218(1)$

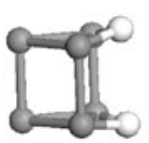

$\mathrm{Ru}_{6} \mathrm{H}_{2}(\mathrm{c})$

$\Delta E=0.0368(5)$

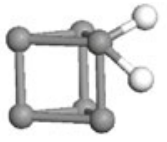

$\mathrm{Ru}_{6} \mathrm{H}_{2}(\mathrm{~d})$

$\Delta E=0.540$ (3)

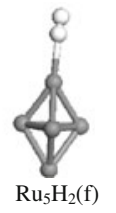

$\Delta E=1.843$ (3)

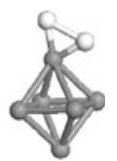

$\mathrm{Ru}_{6} \mathrm{H}_{2}(\mathrm{e})$

$\Delta E=0.637(7)$

Fig. 2 Ground state and some matastable geometries of $\mathrm{Ru}_{n} \mathrm{H}_{2}$ clusters. The dark circles are the $\mathrm{Ru}$ atoms while the white circles are the $\mathrm{H}$ atoms. $\Delta E(\mathrm{eV})$ is the excess energy of an isomer as compared to the energy of the most stable one. The multiplicity is given in parentheses. The symmetry groups of $\mathrm{Ru}_{n} \mathrm{H}_{2}$ clusters are $\mathrm{C}_{1}$ except that of the most stable $\mathrm{Ru}_{n} \mathrm{H}_{2}(n=1,4)\left(\mathrm{C}_{\mathrm{s}}, \mathrm{C}_{2 v}\right)$ 


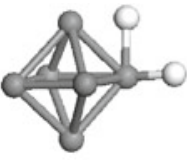

$\mathrm{Ru}_{6} \mathrm{H}_{2}$ (f)

$\Delta E=0.652$ (7)

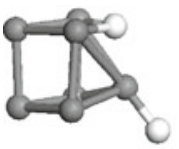

$\mathrm{Ru}_{7} \mathrm{H}_{2}$ (a)

$\Delta E=0(5)$

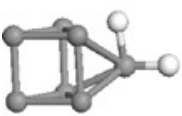

$\mathrm{Ru}_{7} \mathrm{H}_{2}(\mathrm{f})$

$\Delta E=0.706(5)$

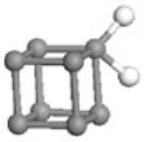

$\mathrm{Ru}_{8} \mathrm{H}_{2}$ (a)

$\Delta E=0(5)$

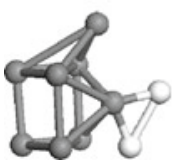

$\mathrm{Ru}_{8} \mathrm{H}_{2}(\mathrm{f})$

$\Delta E=1.577(3)$

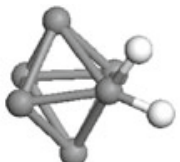

$\mathrm{Ru}_{6} \mathrm{H}_{2}(\mathrm{~g})$

$\Delta E=0.682(5)$

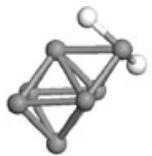

$\mathrm{Ru}_{7} \mathrm{H}_{2}$ (b)

$\Delta E=0.292$ (3)

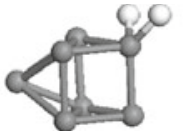

$\mathrm{Ru}_{7} \mathrm{H}_{2}(\mathrm{~g})$

$\Delta E=0.735(5)$

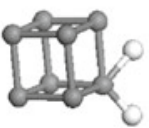

$\mathrm{Ru}_{8} \mathrm{H}_{2}(\mathrm{~b})$

$\Delta E=0.0772(1)$

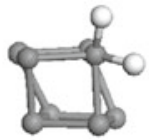

$\mathrm{Ru}_{8} \mathrm{H}_{2}(\mathrm{~g})$

$\Delta E=1.783(3)$

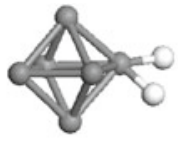

$\mathrm{Ru}_{6} \mathrm{H}_{2}(\mathrm{~h})$

$\Delta E=0.671(7)$

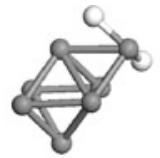

$\mathrm{Ru}_{7} \mathrm{H}_{2}(\mathrm{c})$

$\Delta E=0.304(3)$

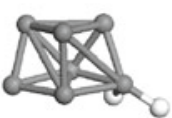

$\mathrm{Ru}_{7} \mathrm{H}_{2}(\mathrm{~h})$

$\Delta E=0.745(3)$

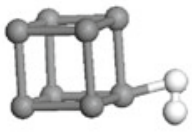

$\mathrm{Ru}_{8} \mathrm{H}_{2}$ (c)

$\Delta E=0.357$ (5)

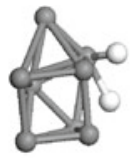

$\mathrm{Ru}_{8} \mathrm{H}_{2}(\mathrm{~h})$

$\Delta E=2.570(5)$

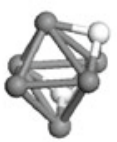

$\mathrm{Ru}_{6} \mathrm{H}_{2}(\mathrm{i})$

$\Delta E=0.700(11)$

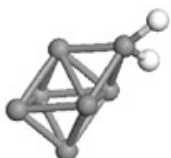

$\mathrm{Ru}_{7} \mathrm{H}_{2}(\mathrm{~d})$

$\Delta E=0.637(3)$

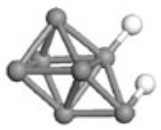

$\mathrm{Ru}_{7} \mathrm{H}_{2}$ (i)

$\Delta E=0.789(5)$

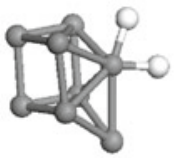

$$
\mathrm{Ru}_{8} \mathrm{H}_{2}(\mathrm{~d})
$$

$\Delta E=1.504$ (3)

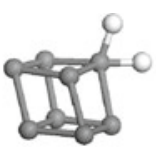

$\mathrm{Ru}_{8} \mathrm{H}_{2}$ (i)

$\Delta E=2.760$ (3)

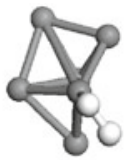

$\mathrm{Ru}_{6} \mathrm{H}_{2}(\mathrm{j})$

$\Delta E=0.792(3)$

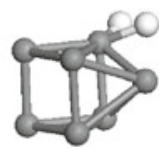

$\mathrm{Ru}_{7} \mathrm{H}_{2}(\mathrm{e})$

$\Delta E=0.689$ (3)

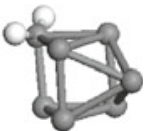

$\mathrm{Ru}_{7} \mathrm{H}_{2}(\mathrm{j})$

$\Delta E=0.834(3)$

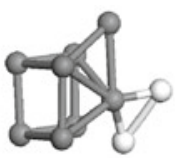

$\mathrm{Ru}_{8} \mathrm{H}_{2}(\mathrm{e})$

$\Delta E=1.544(1)$

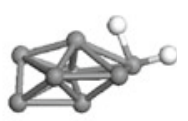

$\mathrm{Ru}_{8} \mathrm{H}_{2}(\mathrm{j})$

$\Delta E=2.892(1)$

Fig. 2 continued

lengths range from 2.493 to $2.598 \AA$ and the shortest from 2.326 to $2.399 \AA$, the longest $\mathrm{R}_{\mathrm{Ru}-\mathrm{H}}$ bond lengths vary from 1.743 to $1.849 \AA$ and the shortest from 1.154 to $1.722 \AA$, and the bond lengths of $\mathrm{H}-\mathrm{H}$ are in the range of 2.320 to $3.419 \AA$, which indicates the $\mathrm{H}_{2}$ is activated.

For $\mathrm{Ru}_{2} \mathrm{H}_{2}$, the ground state is quintet state with $\mathrm{H}-\mathrm{H}$ bond length of $2.173 \AA$. When one $\mathrm{H}$ atom is drawn to one $\mathrm{Ru}$ atom and the other $\mathrm{H}$ atom to the other $\mathrm{Ru}$ atom, the optimized structure (Fig. 2b) is $0.18 \mathrm{eV}$ higher than the ground state. In this structure, both of $\mathrm{Ru}-\mathrm{Ru}-\mathrm{H}$ angles are about $90^{\circ}$. 
Table 1 The longest $\left(\operatorname{Max} \_R_{\mathrm{Ru}-\mathrm{Ru}}, \operatorname{Max} \_R_{\mathrm{Ru}-\mathrm{H}}\right)$ and the shortest $\left(\mathrm{Min} \_R_{\mathrm{Ru}-\mathrm{Ru}}, \mathrm{Min} \_R_{\mathrm{Ru}-\mathrm{H}}\right)$ bond lengths $(\AA)$ and $\mathrm{H}-\mathrm{H}$ bond length $(\AA)\left(R_{\mathrm{H}-\mathrm{H}}\right)$ of the lowest energy structures of $\mathrm{Ru}_{n}$ clusters and $\mathrm{Ru}_{n} \mathrm{H}_{2}$ complexes

\begin{tabular}{|c|c|c|c|c|c|c|c|c|c|}
\hline \multirow[t]{2}{*}{$n$} & \multicolumn{2}{|c|}{ Symmetry } & \multicolumn{2}{|c|}{$\operatorname{Max} \_R_{\mathrm{Ru}-\mathrm{Ru}}$} & \multicolumn{2}{|c|}{ Min $\_R_{\mathrm{Ru}-\mathrm{Ru}}$} & \multirow{2}{*}{$\begin{array}{l}\mathrm{Max} \_R_{\mathrm{Ru}-\mathrm{H}} \\
\mathrm{Ru}_{n} \mathrm{H}_{2}\end{array}$} & \multirow{2}{*}{$\begin{array}{l}\mathrm{Min}_{-} R_{\mathrm{Ru}-\mathrm{H}} \\
\mathrm{Ru}_{n} \mathrm{H}_{2}\end{array}$} & \multirow{2}{*}{$\begin{array}{l}R_{\mathrm{H}-\mathrm{H}} \\
\mathrm{Ru}_{n} \mathrm{H}_{2}\end{array}$} \\
\hline & $\mathrm{Ru}_{n} \mathrm{H}_{2}$ & $\mathrm{Ru}_{n}$ & $\mathrm{Ru}_{n} \mathrm{H}_{2}$ & $\mathrm{Ru}_{n}$ & $\mathrm{Ru}_{n} \mathrm{H}_{2}$ & $\mathrm{Ru}_{n}$ & & & \\
\hline 2 & $\mathrm{C}_{1}$ & $D_{\infty \mathrm{h}}$ & 2.322 & 2.226 & 2.322 & 2.226 & 1.574 & 1.573 & 2.173 \\
\hline 3 & $\mathrm{C}_{1}$ & $\mathrm{C}_{\mathrm{s}}$ & 2.598 & 2.505 & 2.326 & 2.343 & 1.748 & 1.581 & 2.443 \\
\hline 4 & $\mathrm{C}_{\mathrm{s}}$ & $\mathrm{D}_{4 \mathrm{~h}}$ & 2.587 & 2.303 & 2.399 & 2.303 & 1.780 & 1.722 & 2.530 \\
\hline 5 & $\mathrm{C}_{1}$ & $\mathrm{C}_{2 \mathrm{v}}$ & 2.584 & 2.536 & 2.313 & 2.341 & 1.787 & 1.154 & 2.407 \\
\hline 6 & $\mathrm{C}_{1}$ & $\mathrm{C}_{1}$ & 2.542 & 2.459 & 2.325 & 2.342 & 1.795 & 1.661 & 2.571 \\
\hline $7 \mathrm{a}$ & $\mathrm{C}_{1}$ & $\mathrm{C}_{\mathrm{s}}$ & 2.898 & 2.637 & 2.343 & 2.339 & 1.849 & 1.632 & 3.419 \\
\hline $7 b$ & $\mathrm{C}_{1}$ & $\mathrm{C}_{1}$ & 2.610 & 2.609 & 2.398 & 2.338 & 1.810 & 1.750 & 2.988 \\
\hline 8 & $\mathrm{C}_{1}$ & $D_{3 d}$ & 2.493 & 2.370 & 2.336 & 2.363 & 1.743 & 1.633 & 2.320 \\
\hline
\end{tabular}

The most stable structure of $\mathrm{Ru}_{3} \mathrm{H}_{2}$ is a triplet state with $\mathrm{C}_{1}$ symmetry. The structure with a $\mathrm{H}$ is absorbed on the different top site is metastable isomer, its energy is $0.0861 \mathrm{eV}$ higher than that of the lowest energy structure. In case of $\mathrm{Ru}_{4} \mathrm{H}_{2}$, the first and the second $\mathrm{H}$ are both absorbed on the adjacent bridge sites. In the lowest energy structure with a triplet state, the $\mathrm{H}-\mathrm{H}$ distance is $2.530 \AA$, and the $\mathrm{H}_{2}$ is also activated. The structure with two $\mathrm{H}$ atoms absorbed on the top site on the lowest energy structure of $\mathrm{Ru}_{4}$ is metastable isomer, its energy is $0.126 \mathrm{eV}$ higher than that of the lowest energy structure. For $\mathrm{Ru}_{5} \mathrm{H}_{2}$, a square pyramid-based structure in a singlet state is most stable, followed by a singlet, triplet and triplet state with $0.293,0.416$ and $0.457 \mathrm{eV}$ higher in energy, respectively.

In the case of $\mathrm{Ru}_{6} \mathrm{H}_{2}$, the most stable structure is a trigonal prism-based structure in a triplet state, followed by a singlet with $0.0218 \mathrm{eV}$ higher in energy than the most stable structure. The structure with two $\mathrm{H}$ atoms absorbed on the bridge site on the trigonal prism is metastable isomer, the energy is $0.0368 \mathrm{eV}$ higher than that of the lowest energy structure. For $\mathrm{Ru}_{7} \mathrm{H}_{2}$, the most stable structure is a singly capped trigonal prism-based structure in a quintuple state, formed by a hollow site adsorption in which one $\mathrm{H}$ atom sits above the plane of three $\mathrm{Ru}$ atoms and another $\mathrm{H}$ atom locates on the $\mathrm{Ru}$ atom top. The secondary low-lying isomer of $\mathrm{Ru}_{7} \mathrm{H}_{2}$ is a singly capped square bi-pyramid-based structure, which actually corresponds to the third low-lying isomer of pure $\mathrm{Ru}_{7}$ isomer. Other isomers are also presented in Fig. 2. The lowest energy structure of $\mathrm{Ru}_{8} \mathrm{H}_{2}$ is square prism-based structure in a quintet state, the single state is $0.0772 \mathrm{eV}$ higher in energy.

According to above analysis, it is shown that the lowest energy structure of $\mathrm{Ru}_{n} \mathrm{H}_{2}$ can be obtained by adsorbing $\mathrm{H}_{2}$ in the lowest energy and some meta-table isomers of $\mathrm{Ru}_{n}$ clusters. It is interesting to notice that in all cases, the absorbed species does not lead to a rearrangement of the basic cluster, but the hydrogen atom adsorbs to the edge of a $\mathrm{Ru}-\mathrm{Ru}$ bond with the bond lengthening. The longest and the shortest $\mathrm{R}_{\mathrm{Ru}-\mathrm{Ru}}$ (increase with the cluster size overall) as well as $\mathrm{R}_{\mathrm{Ru}-\mathrm{H}}$ vary in small ranges overall. In the $\mathrm{Ru}_{n} \mathrm{H}_{2}, \mathrm{H}-\mathrm{H}$ bond length is about 2.173-3.419 $\AA$. The values 
are larger than the bond length of optimized $\mathrm{H}_{2}(0.748 \AA)$, which indicates the hydrogen molecular is activated.

\section{Relatives Stability and Electronic Properties}

It is known that the relative stability of the different sized cluster can be predicted by calculating the averaged binding energy. The averaged binding energy can be defined as the following formula:

$$
\begin{gathered}
E_{b}\left[\mathrm{Ru}_{n} \mathrm{H}_{2}\right]=\left(-E\left[\mathrm{Ru}_{n} \mathrm{H}_{2}\right]+n E[\mathrm{Ru}]+2 E[\mathrm{H}]\right) /(n+2) \\
E_{b}\left[\mathrm{Ru}_{n}\right]=\left(-E\left[\mathrm{Ru}_{n}\right]+n E[\mathrm{Ru}]\right) / n
\end{gathered}
$$

where $E_{T}\left(\mathrm{Ru}_{n} \mathrm{H}_{2}\right), E_{T}(\mathrm{Ru}), E_{T}(\mathrm{H})$ and $E_{T}\left(\mathrm{Ru}_{n}\right)$ represent the total energies of the $\mathrm{Ru}_{n} \mathrm{H}_{2}, \mathrm{Ru}, \mathrm{H}, \mathrm{Ru}_{n}$ clusters, respectively. The calculated results on the averaged energies for $\mathrm{Ru}_{n} \mathrm{H}_{2}$ and $\mathrm{Ru}_{n}$ clusters are plotted in Fig. 3. When $\mathrm{H}_{2}$ is adsorbed on the $\mathrm{Ru}_{n}$ clusters, the average binding energy is larger than that of $\mathrm{Ru}_{n}$ clusters for $n=2-3$, the average binding energy is smaller than that of $\mathrm{Ru}_{n}$ clusters with cluster size increasing. For $n=2-3$, the $\mathrm{H}$ atom mainly act with $\mathrm{Ru}$ atom. The calculated binding energy (3.577 eV) of $\mathrm{Ru}-\mathrm{H}$ are larger than that of $\mathrm{Ru}-\mathrm{Ru}(3.19 \mathrm{eV})$, which indicates the interaction between $\mathrm{Ru}$ and $\mathrm{H}$ is larger than that of $\mathrm{Ru}-\mathrm{Ru}$. So the averaged energy for $\mathrm{Ru}_{n} \mathrm{H}_{2}$ clusters is larger than that of $\mathrm{Ru}_{n}$. When $n>3$, the $\mathrm{H}$ atoms are mainly bond $\mathrm{Ru}_{n}$ by the weak interaction, which causes the averaged binding energies of $\mathrm{Ru}_{n} \mathrm{H}_{2}$ are smaller than that of $\mathrm{Ru}_{n}$ clusters.

The relative stabilities of these clusters can be better understood by calculating the incremental formation energies, i.e., the second order difference of cluster energies, we defined the $\Delta_{2} E(n)$ as the following formula:

$$
\Delta_{2} E=E(n-1)+E(n+1)-2 E(n)
$$

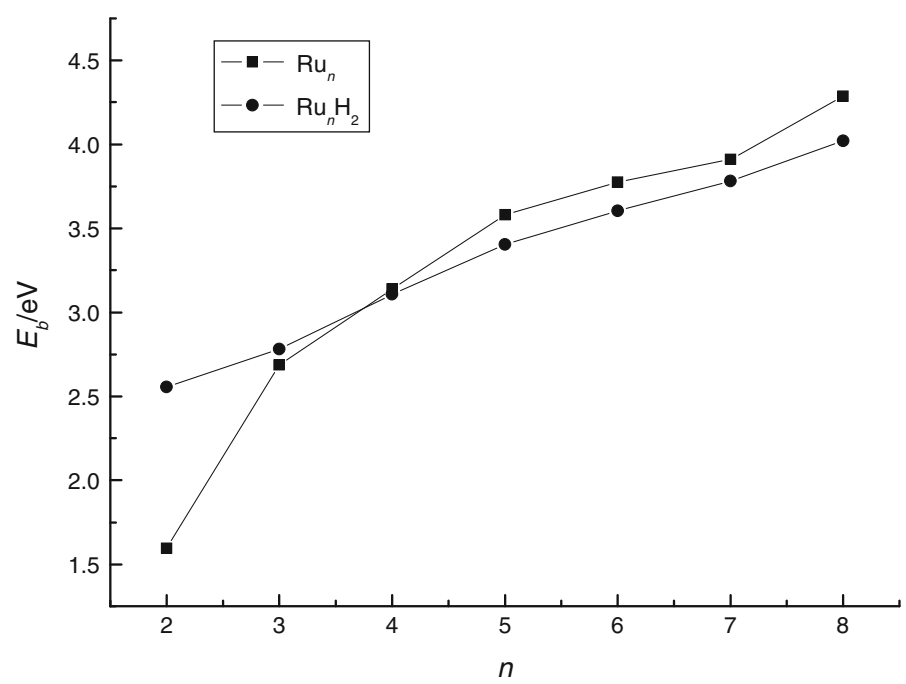

Fig. 3 Binding energy versus cluster size for $\mathrm{Ru}_{n} \mathrm{H}_{2}$ and $\mathrm{Ru}_{n}$ clusters 


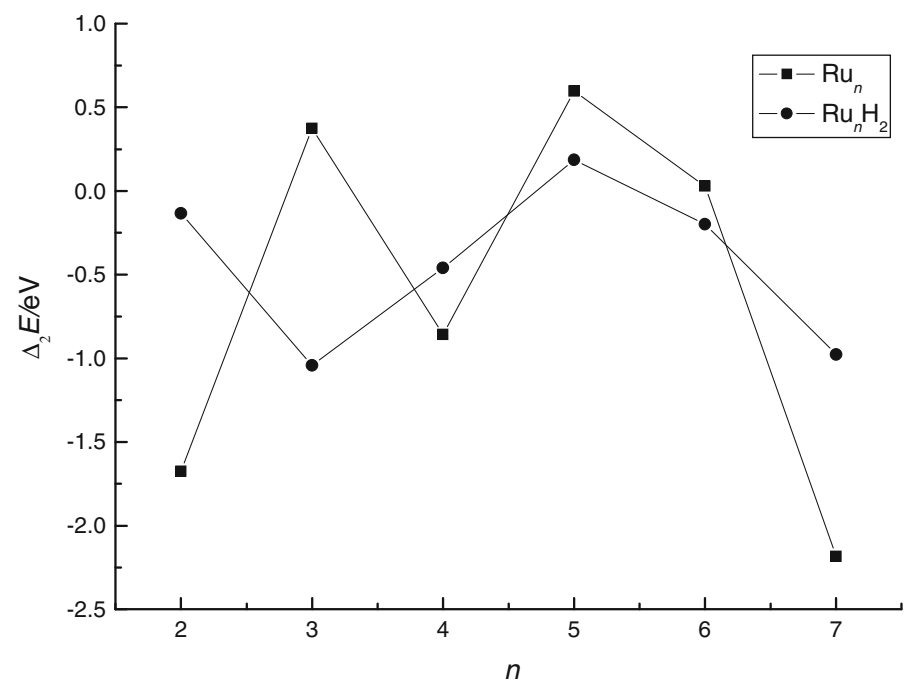

Fig. 4 Second finite difference of the total energies for $\mathrm{Ru}_{n} \mathrm{H}_{2}$ and $\mathrm{Ru}_{n}$ clusters

where $E_{T}(n)$ is the total energy of clusters. The second order difference of cluster energies for $\mathrm{Ru}_{n} \mathrm{H}_{2}$ and $\mathrm{Ru}_{n}$ clusters are all presented in Fig. 4. According to Fig. 4, for $\mathrm{Ru}_{n}$ clusters, the peak values appear at $n=3,5$, it shows apparently that the maximum magic number of the relative stability is $n=3,5$ among investigated $\mathrm{Ru}_{n}$ clusters. For $\mathrm{Ru}_{n} \mathrm{H}_{2}$, particularly high peaks for are found at $n=5$, reflecting that the $\mathrm{Ru}_{5} \mathrm{H}_{2}$ cluster is more stable than its neighboring clusters. The same magic number 5 indicates the effect of $\mathrm{H}$ molecular to the bonding natures of $\mathrm{Ru}_{n}$ clusters is small for $n>3$, which is consistent with the result of the averaged energy.

To measure the strength of the interaction of $\mathrm{H}$ with each of the clusters, we calculated the binding energy of $\mathrm{H}$ to each cluster according to:

$$
E(H)=E\left(\mathrm{Ru}_{n} \mathrm{H}_{2}\right)-E\left(\mathrm{Ru}_{n}\right)-2 E(\mathrm{H})
$$

The calculated binding energies of $\mathrm{H}$ to cluster corresponding to the most energetically favorable chemisorption site are show in Fig. 5, which has a strong dependence on the clusters and increase until $n=2$, and then decrease to the lowest value of $5.847 \mathrm{eV}$ at $n=3$. The bind energies of $\mathrm{H}$ to $\mathrm{Ru}_{n}$ cluster then increase in general as the size of the clusters increase, attaining the local maxima for $n=2,4$ and 7. The larger binding energies of $\mathrm{H}$ to $\mathrm{Ru}_{n}$ cluster $(n=2,3)$ indicate the stronger inaction of $\mathrm{Ru}-\mathrm{H}$, which is consistent with the result of the averaged energy. The values for bind energies of $\mathrm{H}$ to $\mathrm{Ru}_{n}$ cluster are 5.847-7.024 eV, and these is larger than that of $\mathrm{Ru}_{n}$ cluster(1.597-4.295 eV), which also shows the interaction of $\mathrm{Ru}-\mathrm{H}$ is larger than that of $\mathrm{Ru}-\mathrm{Ru}$.

The electronic properties of clusters are discussed by examining the energy gap between the HOMO and LUMO. The HOMO-LUMO gaps for $\mathrm{Ru}_{n} \mathrm{H}_{2}$ and $\mathrm{Ru}_{n}$ clusters of the most stable structure are shown in Fig. 6. As can be seen from Fig. 6, when $\mathrm{H}_{2}$ is absorbed on the $\mathrm{Ru}_{n}$ clusters, the gaps are usually smaller than those of 


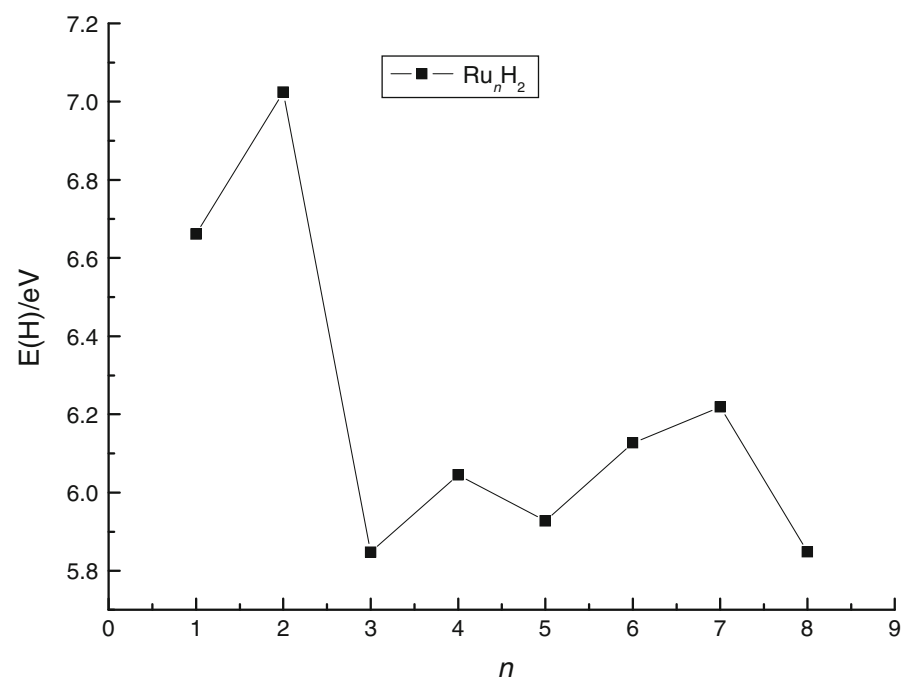

Fig. 5 The binding energies of $\mathrm{H}$ to $\mathrm{Ru}_{n}$ clusters

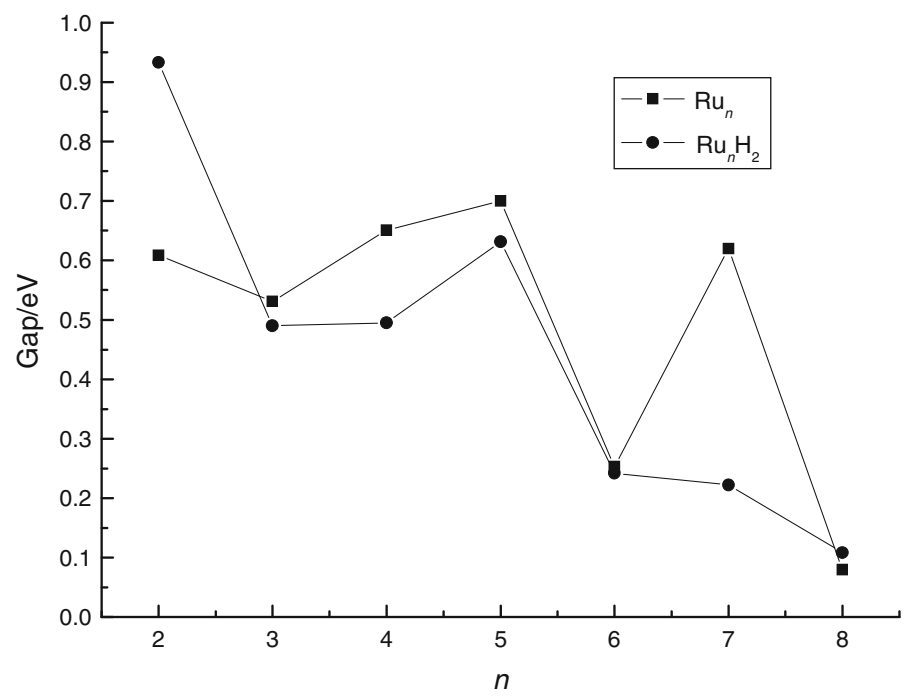

Fig. 6 The HOMO-LUMO Gaps for $\mathrm{Ru}_{n} \mathrm{H}_{2}$ and $\mathrm{Ru}_{n}$ clusters

$\mathrm{Ru}_{n}$ cluster, which indicates that the adsorption of the $\mathrm{H}$ atoms improves the chemical activity of the host clusters in most cases. However, the exceptional case is $n=2$ in which the values of the gap for $\mathrm{Ru}_{2}$ are much larger than those of the $\mathrm{Ru}_{2} \mathrm{H}_{2}$ cluster. Interestingly, the energy gap of $\mathrm{Ru}_{8} \mathrm{H}_{2}$ is close to that of $\mathrm{Ru}_{8}$, which shows that the adsorption has little effect on the chemical activity with $n=8$. Consequently, the $\mathrm{Ru}_{n} \mathrm{H}_{2}$ clusters exhibit remarkable difference in terms of the variation of the energy gap as the $\mathrm{H}$ atoms are adsorbed onto the $\mathrm{Ru}_{n}$ clusters. 


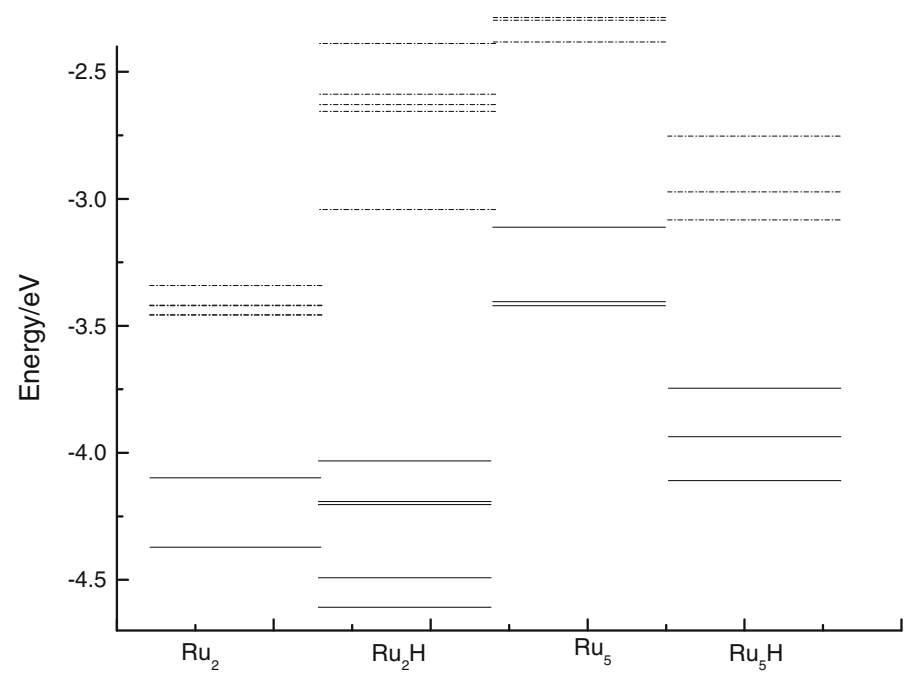

Fig. 7 One electron energy levels in the vicinity of the HOMO and LUMO of the $\mathrm{Ru}_{2} \mathrm{H}_{2}, \mathrm{Ru}_{2}, \mathrm{Ru}_{5} \mathrm{H}_{2}$, $\mathrm{Ru}_{5}$ (The dotted lines represent the LUMO levels, and the solid lines represent HOMO levels)

In light of the particular phenomenon mentioned above, it is necessary to further study the reason about the changing trend of the gap. Meanwhile, the energy level of the molecular orbital for $\mathrm{Ru}_{2}\left(\mathrm{Ru}_{2} \mathrm{H}_{2}\right), \mathrm{Ru}_{5}\left(\mathrm{Ru}_{5} \mathrm{H}_{2}\right)$, which is displayed in Fig. 7 , were investigated to further illustrate the electronic property. The obvious characteristic of the molecular orbital for $\mathrm{Ru}_{2}$ is the appearance of the degeneracy of the energy level in the vicinity of the HOMO-LUMO. With the $\mathrm{H}$ atoms being adsorbed onto the $\mathrm{Ru}_{2}$ cluster, the degenerate energy level is completely disappeared. Moreover, compared with $\mathrm{Ru}_{2}$, the energy level of the LUMO for $\mathrm{Ru}_{2} \mathrm{H}_{2}$ is elevated strongly (about $0.938 \mathrm{eV}$ ). In case of $\mathrm{Ru}_{5}$, there is also degenerate orbital in the vicinity of HOMO-LUMO. However, the HOMO and LUMO for $\mathrm{Ru}_{5} \mathrm{H}_{2}$ both consist of two degenerate orbitals, respectively. When $\mathrm{H}$ atoms are adsorbed onto the $\mathrm{Ru}_{5}$ cluster, the LUMO of $\mathrm{Ru}_{5} \mathrm{H}_{2}$ is also reduced (about $0.669 \mathrm{eV}$ ) in comparison to $\mathrm{Ru}_{5}$. Apparently, the similar behavior of the reduction of the LUMO for $n=3,4,6,7$, results in the decrease of their energy gap.

In Fig. 8, we give the adsorption energy for $\mathrm{Ru}_{n} \mathrm{H}_{2}$ complex. The absorbed energy can be defined as the following formula:

$$
E_{\text {adv }}=E_{\mathrm{H}_{2}}+E_{\text {cluster }}-E_{\text {cluster }+\mathrm{H}_{2}}
$$

It is well-known that the chemisorption energies can be used to quantitatively describe the reactivity of $\mathrm{H}_{2}$ on the $\mathrm{Ru}_{n}$ clusters. The calculated chemisorption energies corresponding to the most energetically favorable chemisorption site are show in Fig. 8, which has a strong dependence on the clusters and increase until $n=2$, and then decrease to the lowest value of $1.182 \mathrm{eV}$ at $n=3$. The chemisorption energy then increase in general as the size of the clusters increase, attaining the local maxima for $n=2,4$ and 7. The present results show the $\mathrm{Ru}_{3}$ cluster is of high inertness with respect to the $\mathrm{H}_{2}$ chemisorption, while $\mathrm{Ru}_{2}, \mathrm{Ru}_{4}$, and $\mathrm{Ru}_{7}$ clusters are of high activity with respect to the $\mathrm{H}_{2}$ chemisorption. Note that 


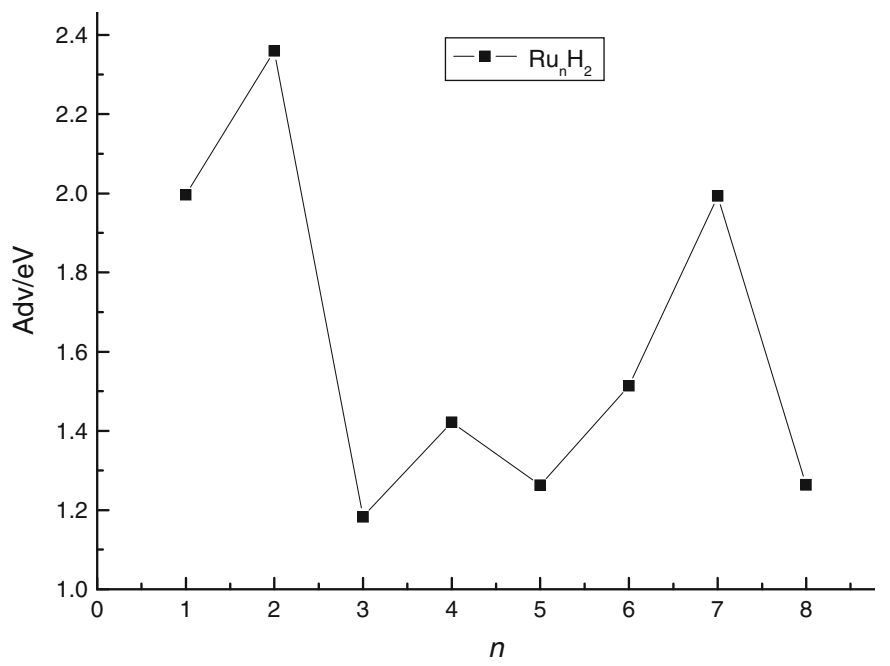

Fig. 8 The absorbed energy $\left(\mathrm{A}_{\mathrm{dv}}\right)$ for $\mathrm{Ru}_{n} \mathrm{H}_{2}$

Dhilip Kumar et al. [46] also calculated that the $\mathrm{H}_{2}$ chemisorption energy was found to be the lowest for the stable $\mathrm{Ti}_{7}$ cluster but highest for the most stable cluster $\mathrm{Ti}_{13}$. Experimental measurements indicate that when $\mathrm{Ti}_{13}$ cluster is added as a catalyst to increase the reaction rate of hydrogenation and de hydrogenation processes in alanates [47]. Whether the $\mathrm{Ru}_{2}$ is added as a catalyst to increase the reaction rate of hydrogenation and de hydrogenation processes is to be studied further theoretically and experimentally.

The charge transfer from the $\mathrm{Ru}_{n}$ cluster to $\mathrm{H}_{2}$ molecule is an essential factor to determine the $\mathrm{H}_{2}$ adsorption behavior on $\mathrm{Ru}_{n}$ clusters. Here we give the charge populations from natural population analysis on $\mathrm{H}$ atoms (In Fig. 9). According to the NPA charge population, electron transfer will occur from $\mathrm{H}$ atoms to $\mathrm{Ru}_{n}$ clusters. The electron transferred number between $\mathrm{H}$ atoms and $\mathrm{Ru}_{n}$ clusters is about $0.0475-0.158 \mathrm{e}$, this means the interaction between $\mathrm{H}$ atoms and $\mathrm{Ru}_{n}$ clusters is small. When $n=2,7$, the charges from $\mathrm{H}$ atoms to $\mathrm{Ru}_{n}$ clusters are more, which is consist with the large absorbed energy in these clusters. For $\mathrm{Ru}_{6} \mathrm{H}_{2}$, the charges from $\mathrm{H}$ atoms to $\mathrm{Ru}_{n}$ clusters are smallest among $\mathrm{Ru}_{n} \mathrm{H}_{2}$ complex, this indicates the adsorption has little effect on $\mathrm{Ru}_{6}$, which is the reason that the energy gap of $\mathrm{Ru}_{6} \mathrm{H}_{2}$ is close to that of $\mathrm{Ru}_{6}$.

\section{Magnetic Properties}

Figure 10 gives the spin magnetic moment of pure and hydrogenated clusters. For $\mathrm{Ru}_{3}$, the $\mathrm{H}$ atoms decrease the spin magnetic moment. For $\mathrm{Ru}_{2}, \mathrm{Ru}_{4}$ and $\mathrm{Ru}_{7}$, the $\mathrm{H}$ atoms increase the spin magnetic moment. In case of $\mathrm{Ru}_{n}(n=5,6,8)$, the $\mathrm{H}$ atoms have little impact on the spin magnetic moment. In a previous paper, Fournier et al. have proposed $\mathrm{H}_{2}$ affect on spin magnetic moment of transitional metal clusters can be understood within a simple model $[48,49]$. The change in moment is related to 


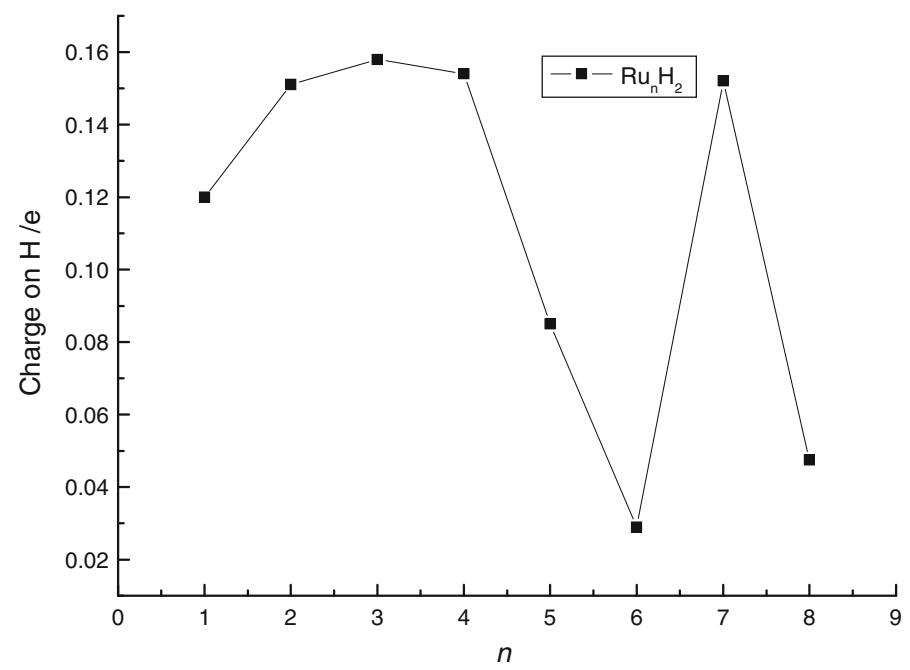

Fig. 9 The averaged charge on the $\mathrm{H}$ atoms for $\mathrm{Ru}_{n} \mathrm{H}_{2}$

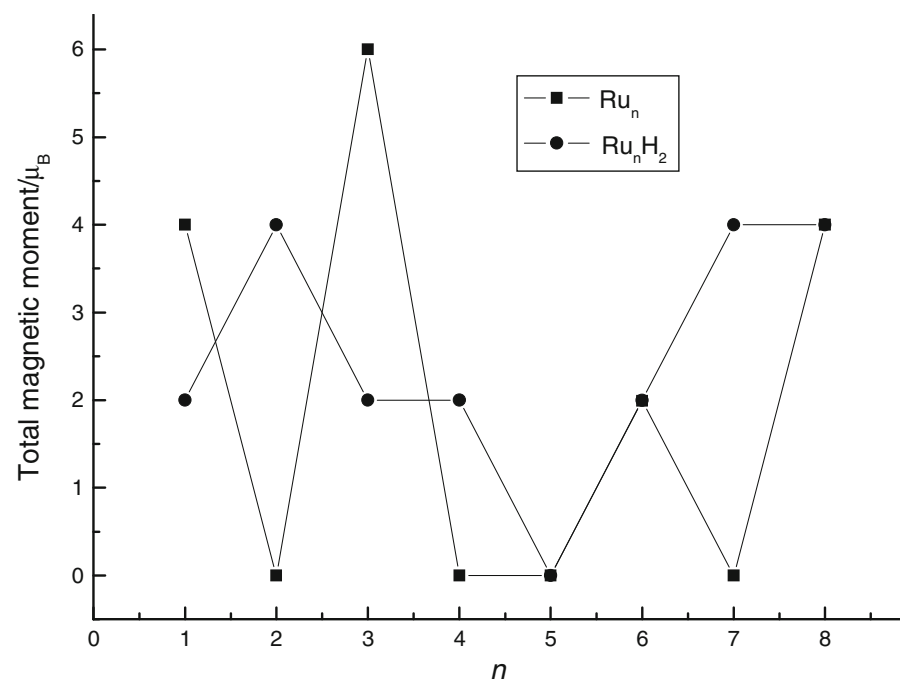

Fig. 10 The total magnetic moment for $\mathrm{Ru}_{n} \mathrm{H}_{2}$ and $\mathrm{Ru}_{n}$ clusters

the location of the lowest unoccupied orbital of the preceding cluster. The $\mathrm{H}$ atom can be considered as a proton and an electron. The additional electron goes to the spin state with lowest LUMO while the proton is screened by the $d$-states of the neighboring Ru sites. This however, is not the only consideration. If the LUMO of the preceding cluster belongs to the minority manifold (spin down) and the LUMO of majority (spin up) is only slightly higher, the additional electron may still go to majority manifold since the exchange coupling could lead to a rearrangement of the manifolds. To put it simply, it is the difference, $\delta \mathrm{E}$, between the LUMO of the 
Table 2 The HOMO and LUMO levels (hartrees) of the majority and minority spin states and $\delta E(\mathrm{eV})$ in $\mathrm{Ru}_{n}$ and $\mathrm{Ru}_{n} \mathrm{H}_{2}$ clusters

\begin{tabular}{|c|c|c|c|c|c|}
\hline \multirow[t]{2}{*}{ Cluster } & \multicolumn{2}{|l|}{ Majority } & \multicolumn{3}{|l|}{ Minority } \\
\hline & HOMO & LUMO & HOMO & LUMO & $\delta \mathrm{E}$ \\
\hline $\mathrm{Ru}$ & -0.165049 & 0.028365 & -1.68319 & -0.127363 & -4.235 \\
\hline $\mathrm{RuH}_{2}$ & -0.192468 & -0.085494 & -0.175048 & -0.126172 & -1.105 \\
\hline $\mathrm{Ru}_{2}$ & -0.152441 & -0.130001 & -0.152425 & -0.130039 & -0.00103 \\
\hline $\mathrm{Ru}_{2} \mathrm{H}_{2}$ & -0.155393 & -0.099403 & -0.149725 & -0.115411 & -0.523 \\
\hline $\mathrm{Ru}_{3}$ & -0.139542 & -0.096201 & -0.119653 & -0.100129 & -0.107 \\
\hline $\mathrm{Ru}_{3} \mathrm{H}_{2}$ & -0.145644 & -0.127625 & -0.145781 & -0.118462 & 0.249 \\
\hline $\mathrm{Ru}_{4}$ & -0.131310 & -0.107383 & -0.13110 & -0.107383 & 0.000 \\
\hline $\mathrm{Ru}_{4} \mathrm{H}_{2}$ & -0.130210 & -0.091910 & -0.121221 & -0.103016 & -0.302 \\
\hline $\mathrm{Ru}_{5}$ & -0.117938 & -0.092193 & -0.117938 & -0.092193 & 0.000 \\
\hline $\mathrm{Ru}_{5} \mathrm{H}_{2}$ & -0.140009 & -0.116785 & -0.14009 & -0.116785 & 0.000 \\
\hline $\mathrm{Ru}_{6}$ & -0.112439 & -0.103139 & -0.116246 & -0.102145 & 0.0270 \\
\hline $\mathrm{Ru}_{6} \mathrm{H}_{2}$ & -0.130284 & -0.121382 & -0.135251 & -0.119575 & 0.0492 \\
\hline $\mathrm{Ru}_{7}$ & -0.138811 & -0.115984 & -0.138843 & -0.116027 & -0.00117 \\
\hline $\mathrm{Ru}_{7} \mathrm{H}_{2}$ & -0.138365 & -0.125574 & -0.137621 & -0.126381 & -0.0220 \\
\hline $\mathrm{Ru}_{8}$ & -0.127748 & -0.124811 & -0.147696 & -0.108937 & 0.432 \\
\hline $\mathrm{Ru}_{8} \mathrm{H}_{2}$ & -0.139109 & -0.135132 & -0.158438 & -0.125275 & 0.268 \\
\hline
\end{tabular}

majority and the minority spin manifolds that controls the change in moment. When this quantity is positive, one expects the moment to increase. On the other hand, when this quantity is highly negative, an addition of $\mathrm{H}$ would lead to a decrease in the magnetic moment. To show this correlation, we list in Table 2, the HOMO and LUMO of all the clusters. Note that when $\delta \mathrm{E}$ is less than $0 \mathrm{eV}$, the spin magnetic moment does decrease upon addition of $\mathrm{H}_{2}$. When $\delta \mathrm{E}$ is close to or more than $0 \mathrm{eV}$, the spin magnetic moment increases or varies little upon addition of $\mathrm{H}_{2}$.

According to above analysis, it again identify the difference between the LUMO of the majority and the minority spin manifolds that controls the change in magnetic moment, the change rule in magnetic moment is different when $\mathrm{H}_{2}$ is adsorbed on different transitional metal clusters. The model may be predict the change in magnetic moment when small molecular is adsorbed on the transitional metal clusters.

\section{Conclusions}

We have presented a systematic study on the interaction of the hydrogen atoms with the small $\mathrm{Ru}_{n}$ clusters in the size range of two to eight $\mathrm{Ru}$ atoms. The lowest energy structures of $\mathrm{Ru}_{n} \mathrm{H}_{2}$ clusters can be obtained by substituting $\mathrm{H}_{2}$ in the lowest energy and some meta-stable isomers of $\mathrm{Ru}_{n}$ clusters. For $n>2$, three different adsorption 
patterns are found for the $\mathrm{Ru}_{n} \mathrm{H}_{2}$ complexes: One $\mathrm{H}$ atom binds to the $\mathrm{Ru}$ top site, and another $\mathrm{H}$ binds to the bridge site for $n=3,5,6,8$; bridge site adsorption for $n=4$; hollow site and top site adsorption for $n=7$. The adsorption energies display oscillation and reach the peak at $n=2,4,7$, implying their high chemical reactivity. The small electron transferred number between $\mathrm{H}$ atoms and $\mathrm{Ru}_{n}$ clusters indicates the interaction between $\mathrm{H}$ atoms and $\mathrm{Ru}_{n}$ clusters is small. It is interesting to note that in all cases, the absorbed species does not lead to a rearrangement of the basic cluster. When $\mathrm{H}_{2}$ is absorbed on the $\mathrm{Ru}_{n}$ clusters, the chemical activity of corresponding clusters is dramatically increased. The present studies show that the $\mathrm{H}$ absorption can lead to an oscillatory behavior of the magnetic moments. This behavior is rooted in the electronic structure of the preceding cluster and the changes in the magnetic moment are indicative of the relative ordering of the majority and minority LUMO's. The second order difference indicates 5 is magic number in $\mathrm{Ru}_{n} \mathrm{H}_{2}$ and $\mathrm{Ru}_{n}$ clusters.

Acknowledgments This project supported by foundation start up for high level talents of shihezi university, china (Grant No: RCZX200747).

Open Access This article is distributed under the terms of the Creative Commons Attribution Noncommercial License which permits any noncommercial use, distribution, and reproduction in any medium, provided the original author(s) and source are credited.

\section{References}

1. J. T. Stuckless, C. E. Wartnaby, N. Al-Sarraf, St. J. B. Dixon-Warren, M. Kovar, and D. A. King (1997). J. Chem. Phys. 106, 2012.

2. R. Schaub, P. Thostrup, N. Lopez, E. Laegsgaard, I. Stensgaard, J. K. Nørskov, and F. Besenbacher (2001). Phys. Rev. Lett. 87, 266104.

3. K. C. Prince, G. Paolucci, and A. M. Bradshaw (1986). Surf. Sci. 175, 101.

4. D. A. Outka, J. Stöhr, W. Jark, P. Stevens, J. Solomon, and R. J. Madix (1987). Phys. Rev. B 35, 4119.

5. B. E. Salisbury, W. T. Wallace, and R. L. Whetten (2000). Chem. Phys. 262, 131.

6. L. Holmgren and A. Rosén (1999). J. Chem. Phys. 110, 2629.

7. M. Balooch, M. J. Cardillo, D. R. Miller, and R. E. Stickney (1978). Surf. Sci. 46, 358.

8. B. E. Hayden and C. L. A. Lamont (1989). Phys. Rev. Lett. 63, 1823.

9. A. C. Luntz, J. K. Brown, and M. D. Williams (1990). J. Chem. Phys. 93, 5240.

10. C. T. Rettner, D. J. Auerbach, and H. A. Michelsen (1992). Phys. Rev. Lett. 68, 1164.

11. R. Linke, U. Schneider, H. Busse, C. Becker, U. Schroeder, G. R. Castro, and K. Wandelt (1994). Surf. Sci. 307, 407.

12. U. Schneider, H. Busse, R. Linke, G. R. Castro, and K. Wandelt (1994). J. Vac. Sci. Technol. A 12, 2069.

13. P. M. Holmblad, J. H. Larsen, and I. Chorkendorff (1996). J. Chem. Phys. 104, 7289.

14. A. T. Pasteur, St. J. Dixon-Warren, Q. Ge, and D. A. King (1997). J. Chem. Phys. 106, 8896.

15. B. Fruberger, J. Eng Jr., and J. G. Chen (1997). Catal. Lett. 45, 85.

16. A. Berces, P. A. Hackett, L. Lian, S. A. Mitchell, and D. M. Rayner (1998). J. Chem. Phys. 108, 5476.

17. P. Samson, A. Nesbitt, B. E. Koel, and A. Hodgson (1998). J. Chem. Phys. 109, 3255.

18. K. Moritani, M. Okada, M. Nakamura, T. Kasai, and Y. Murata (2001). J. Chem. Phys. 115, 9947.

19. C. EibI and A. Winkler (2002). J. Chem. Phys. 117, 834.

20. M. F. Somers, D. A. McCormack, G. J. Kroes, R. A. Olsen, E. J. Baerends, and R. C. Mowrey (2002). J. Chem. Phys. 117, 6673. 
21. P. Ravindran, P. Vajeeston, R. Vidya, A. Kjekshus, and H. Fjellvag (2002). Phys. Rev. Lett. 89, 106403.

22. A. Pramann (2001). J. Chem. Phys. 115, 5404.

23. E. Poulain, J. I. Benítez, S. Castillo, V. Bertin, and A. Cruz (2004). J. Mol. Struct. (Theochem.) 709, 67.

24. H. Str $\varphi$ msnes, S. Jusuf, B. Schimmelpfenning, U. Wahlgren, and O. Gropen (2001). J. Mole. Struct. 137, 567.

25. R. L. Whetten, D. M. Cox, D. J. Trevor, and A. Kaldor (1985). Phys. Rev. Lett. 54, 1494.

26. M. B. Knickelbein, G. M. Koretsky, K. A. Jackson, M. R. Pederson, and Z. Hajnal (1998). J. Chem. Phys. 109, 10692.

27. M. B. Knickelbein (1999). Ann. Rev. Phys. Chem. 50, 79.

28. S. P. Liu, C. Hao, S. M. Li, and Z. X. Wang (2009). Appl. Sur. Sci. 225, 4232.

29. C. Crisafulli, S. Scirè, S. Minicò, and L. Solarino (2002). Appl Catal A: General 225, 1.

30. R. Berthoud, P. Délichère, D. Gajan, W. Lukens, K. Pelzer, J. M. Basset, J. P. Candy, and C. Copéret (2008). J. Catal. 260, 387.

31. W. Q. Zheng, J. Zhang, H. Y. Xu, and W. Z. Li (2007). Catal. Lett. 119, 311.

32. X. Y. Tu, D. W. Wang, and X. M. Chen (2004). Precious Metals 25, 15.

33. G. Suss-Fink (2000). J. Organomet. Chem. 609, 196.

34. R. D. Adams, B. Captain, C. Beddie, and M. B. Hall (2007). J. Am. Chem. Soc. 129, 986.

35. R. D. Adams, B. Captain, and L. Zhu (2007). J. Am. Chem. Soc. 129, 2454.

36. R. D. Adams, B. Captain, M. D. Smith, C. Beddie, and M. B. Hall (2007). J. Am. Chem. Soc. 129, 5981.

37. R. D. Adams, E. M. Boswell, M. B. Hall, and X. Yang (2008). Organometallics 27, 4938.

38. I. Rosal, L. Truflandier, R. Poteau, and I. C. Gerber (2011). J. Phys. Chem. C 115, 2169.

39. T. Gutmann, B. Walaszek, Y. P. Xu, M. Wächtler, I. del Rosal, A. GrOnberg, R. Poteau, R. Axet, G. Lavigne, B. Chaudret, H. H. Limbach, and G. Buntkowsky (2010). J. Am. Chem. Soc. 132, 11759.

40. B. Delley (1990). J. Chem. Phys. 92, 508.

41. R. S. Mulliken (1955). J. Chem. Phys. 23, 1841.

42. J. Andzelm, E. Radzio, and D. R. Salahub (1985). J. Chem. Phys. 83, 4573.

43. H. Wang, Y. Liu, H. Haouari, R. Craig, J. R. Lombardi, and D. M. Lindsay (1997). J. Chem. Phys. 106, 6534.

44. D. R. Lide (ed.) CRC handbook of chemistry and physics, 79th (CRC Press, New York, 1998), pp. $9-80$.

45. X. Y. Tu, D. W. Wang, and X. M. Chen (2004). Chin. J. Struct. Chem. 23, 940.

46. T. J. Dhilip Kumar, P. F. Weck, and N. Balakrishnan (2007). J. Phys. Chem. C 111, 7494.

47. M. Fichtner, O. Fuhr, O. Kircher, and J. Rothe (2003). Nanotechonlogy 14, 778.

48. R. Fournier and D. R. Salahub (1990). Surf. Sci. 238, 330.

49. C. Ashman, S. N. Khanna, and M. R. Pederson (2003). Chem. Phys. Lett. 368, 257. 